Light control and image transmission through photonic lattices with engineered

\title{
Zhigang Chen
}

SAN FRANCISCO STATE UNIVERSITY

05/05/2015

Final Report

DISTRIBUTION A: Distribution approved for public release.

Air Force Research Laboratory

AF Office Of Scientific Research (AFOSR)/ RTB

Arlington, Virginia 22203

Air Force Materiel Command 


\section{REPORT DOCUMENTATION PAGE}

Form Approved

OMB No. 0704-0188

The public reporting burden for this collection of information is estimated to average 1 hour per response, including the time for reviewing instructions, searching existing data sources, gathering and maintaining the data needed, and completing and reviewing the collection of information. Send comments regarding this burden estimate or any other aspect of this collection of information, including suggestions for reducing the burden, to Department of Defense, Executive Services, Directorate (0704-0188). Respondents should be aware that notwithstanding any other provision of law, no person shall be subject to any penalty for failing to comply with a collection of information if it does not display a currently valid OMB control number.

PLEASE DO NOT RETURN YOUR FORM TO THE ABOVE ORGANIZATION.

\begin{tabular}{l|l} 
1. REPORT DATE (DD-MM-YYYY) & 2. REPORT TYPE
\end{tabular}

\begin{tabular}{l|l}
$21-05-2015$ & 2. REPORT TYPE \\
Final Performance
\end{tabular}

\section{TITLE AND SUBTITLE}

Light control and image transmission through photonic lattices with engineered coupling

\section{AUTHOR(S)}

Zhigang Chen
3. DATES COVERED (FrOM - TO)

01-04-2012 to 31-03-2015 5a. CONTRACT NUMBER

5b. GRANT NUMBER

FA9550-12-1-0111

5c. PROGRAM ELEMENT NUMBER

5d. PROJECT NUMBER

5e. TASK NUMBER

5f. WORK UNIT NUMBER

\section{PERFORMING ORGANIZATION NAME(S) AND ADDRESS(ES)}

SAN FRANCISCO STATE UNIVERSITY

1600 HOLLOWAY AVE BUILDING NAD ROOM 358C

SAN FRANCISCO, CA 941321722 US

\section{SPONSORING/MONITORING AGENCY NAME(S) AND ADDRESS(ES)}

AF Office of Scientific Research

875 N. Randolph St. Room 3112

Arlington, VA 22203
8. PERFORMING ORGANIZATION REPORT NUMBER

10. SPONSOR/MONITOR'S ACRONYM(S) AFOSR

11. SPONSOR/MONITOR'S REPORT NUMBER(S)

\section{DISTRIBUTION/AVAILABILITY STATEMENT}

A DISTRIBUTION UNLIMITED: PB Public Release

\section{SUPPLEMENTARY NOTES}

\section{ABSTRACT}

The objective of this project is to develop research programs in photonic lattices that are at the frontier of nonlinear optics/photonics for fundamental understandings in scientific knowledge as well as for possible applications of direct interest to the Air Force. The proposed studies include mainly beam control in engineered photonic lattices, Tamm and Shockley-like edge states and topological surface states in 2D honey-comb lattices (photonic graphene), and light localization and transport in disordered lattices. Apart from these proposed studies, the project made additional efforts on design and control of self-accelerating beams and nonlinear enhanced transmission in synthetic colloidal nanosuspensions. These studies will advance the knowledge in several interdisciplinary areas such as nonlinear optics, condensed matter physics, atmospheric sciences, and will also have many application potentials.

15. SUBJECT TERMS

Light Propagation, Nonlinear Crystals, Nonlinear Schrodinger Equation

16. SECURITY CLASSIFICATION OF:

\begin{tabular}{|c|c|c|}
\hline a. REPORT & b. ABSTRACT & c. THIS PAGE \\
& $U$ & $U$ \\
& & \\
\hline
\end{tabular}

\section{LIMITATION OF 1 18. NUMBER 1 19a. NAME OF RESPONSIBLE PERSON} ABSTRACT

UU

OF Zhigang Chen

PAGES 19b. TELEPHONE NUMBER (Include area code) 415-338-3876 
FINAL PERFORMANCE REPORT (APRIL 2015)

\author{
Zhigang Chen \\ San Francisco State University \\ zhigang@sfsu.edu
}

Title: Light Control and Image Transmission through Photonic Lattices with Engineered Coupling

Grant \#: $\quad$ AFOSR \# FA9550-12-1-0111

Program Manager: Dr. Arje Nachman

Reporting Period: $\quad$ APRIL 2012 - MARCH 2015

\begin{abstract}
:
The objective of this project is to develop research programs in photonic lattices that are at the frontier of nonlinear optics/photonics for fundamental understandings in scientific knowledge as well as for possible applications of direct interest to the Air Force. The proposed studies include mainly beam control in engineered photonic lattices, Tamm and Shockley-like edge states and topological surface states in 2D honey-comb lattices ("photonic graphene"), and light localization and transport in disordered lattices. Apart from these proposed studies, the project made additional efforts on design and control of self-accelerating beams and nonlinear enhanced transmission in synthetic colloidal nanosuspensions. These studies will advance the knowledge in several interdisciplinary areas such as nonlinear optics, condensed matter physics, atmospheric sciences, and will also have many application potentials.

In this project, the optical induction technique has been employed to establish various specially designed photonic structures as a workbench for investigating some fundamental wave phenomena. These include, for example, image transmission through periodic media based on light tunneling inhibition, nontrivial surface (edge) states in honeycomb lattices, and accelerating diffraction-free beams and optical analog of Wannier-Stark beams in engineered photonic lattices. Although performed in a simple optical setting of reconfigurable photonic structures, much of our proposed work will have direct impact on other areas of sciences, ranging from solid state physics to atom physics such as Bose-Einstein condensates trapped in periodic potentials. As an example, the electronic edge states were known for decades, but such fundamental phenomena have recently attracted growing interest in optics that has led to the successful demonstration of photonic topological insulators. Indeed, this project shows that there is much potential to use optical systems as photonic simulators for studying complex classical and quantum phenomena.

Our previous work on nonlinear optics/photonics research was supported by a research grant from AFOSR (\#FA9550-09-1-0474; Point of contact: Dr. Nachman), which ended in November, 2011. Earlier progress and success has been detailed in the final technical report submitted for that project, and has also been presented at the annual workshops on Nonlinear Optics organized by Dr. Nachman. This past 3-year contract (\#FA9550-12-1-0111; Point of contact: Dr. Nachman, AFOSR/RSE) started in April 2012. During the three project years, the PI's group published about 40 referred papers that credited AFOSR, and about a dozen students at SFSU have participated in this funded project. Our work has been featured in Optics \& Photonic News as one of the major
\end{abstract}


breakthroughs in optics of the year, has been cited frequently in literature. The results have also been presented in several invited and contributed talks at leading professional meetings and workshops. Here, we provide a summary report for our accomplishments.

\section{Highlight of Major Efforts and Accomplishments:}

In the last three year, we have successfully demonstrated several novel phenomena related to spatial beam dynamics and control of light in photonic bandgap structures, which led to high-level publications in prestigious journals. These include for example eliminating soliton transverse instability in one-dimensional lattices, inducing deep penetration and transparency in highly scattering media, controlling self-accelerating beams in free-space as well as in photonic lattices, and unveiling unconventional edge states in photonic graphene. In particular, we observed new edge states in zigzag and bearded edges of photonic graphene (published in Nature Materials), deep penetration of light through scattering media (published in Physics Review Letters and Nano Letters, and featured in Nature Photonics and Physics), and large-bending angle nonparaxial accelerating beams along curved trajectories (reported in a number of news media as well as featured in PRL cover and Optics and Photonics News). We are happy to report that we have made significant progress in this funded project with about 40 scientific research papers published in top-rated journals. (See attached list of publications that acknowledged the support from AFOSR). In addition, several students have actively involved and contributed significantly in this project, through which they were also trained in scientific areas of interest to Air Force. Close collaborations have been maintained with other principal investigators currently supported by AFOSR including Profs. Christodoulides, Ablowitz, and Yang. The following is a brief summary of our major accomplishments.

1. Edge states in photonic graphene:

We developed a simple technique to optical induce 'photonic graphene' lattices and to observe edge states in these lattices. Specifically, we used the optical equivalent of graphene-a photonic honeycomb lattice - to study the edge states and their properties. We directly imaged the edge states on both the zigzag and bearded edges of this photonic graphene, measured their dispersion properties, and most importantly, found a new type of edge state: one residing on the bearded edge that has never been predicted or observed. Our work led to a number of subsequent discoveries in "photonic graphene" including the demonstration of optical topological insulator.

2. Wannier-Stark beams and accelerating diffractionless beams in photonic lattices.

We generated optical beams analogous to the Wannier-Stark states in semiconductor superlattices and observe that the two main lobes of the WS beams self-bend (accelerate) along two opposite trajectories in a uniform one dimensional photonic lattice. We also showed that only a unique class of zdependent lattices can support a true accelerating diffractionless beam. Accelerating lattice solitons, autofocusing beams and accelerating bullets in optical lattices are systematically examined. 
3. Deep penetration of light needles through highly scattering media

We have demonstrated two types of soft-matter systems with tunable optical nonlinearities - the dielectric and metallic colloidal suspensions. In both systems, we can alter at will the nonlinear light-matter interactions in order to overcome the effects of diffraction and scattering, hence achieving deep penetration of light needle through the colloid.

4. Linear and nonlinear nonparaxial self-accelerating beams

We studied linear and nonlinear self-accelerating beams propagating along circular trajectories beyond the paraxial approximation. Such nonparaxial accelerating beams are exact solutions of the Helmholtz equation, preserving their shapes during propagation even under nonlinearity. We generate experimentally and observe directly these large-angle bending beams in colloidal suspensions of polystyrene nano-particles.

In addition, we demonstrated both theoretically and experimentally nonparaxial Mathieu and Weber accelerating beams, generalizing the concept of previously found accelerating beams. We showed that such beams bend into large angles along circular, elliptical, or parabolic trajectories but still retain nondiffracting and self-healing capabilities. Not only do generalized nonparaxial accelerating beams open up many possibilities of beam engineering for applications, but the fundamental concept developed here can be applied to other linear wave systems in nature, ranging from electromagnetic and elastic waves to matter waves.

This part of the work has merited a few papers published in Optics Letters and Physical Review Letters.

5. Elimination of transverse instability in stripe solitons by one-dimensional lattices

In collaboration with AFOSR contractor Dr. J. Yang, we demonstrate theoretically and experimentally that the transverse instability of coherent soliton stripes can be greatly suppressed or totally eliminated when the soliton stripes propagate in a one-dimensional photonic lattice under self-defocusing nonlinearity. Both schemes and demonstrations were published in Optics Letters.

6. Self-accelerating Bessel-like optical beams along arbitrary trajectories

In collaboration with AFOSR contractor Dr. Christodoulides, we proposed and experimentally demonstrated self-accelerating Bessel-like optical beams propagating along arbitrary trajectories in free space. With computer generated holography, such beams are designed to follow different controllable trajectories while their main lobe transverse profiles remain nearly invariant and symmetric. Examples include parabolic, snake-like, hyperbolic, hyperbolic secant, and even three-dimensional spiraling trajectories. The selfhealing property of such beams is also demonstrated. This new class of optical beams can 
be considered as a hybrid between accelerating and non-accelerating nondiffracting beams that may find a variety of applications.

In addition, we demonstrated optical trapping and guiding micro-particles, aerosols and bacteria with a variety of specially designed optical beams, including rotating tweezers, optical bottles, and auto-focusing beams. This part of work has been published in several Optics Letter papers.

\section{Interactions/Collaborations:}

Participation of students and postdoc researchers in the proposed research

The P.I. has actively engaged students, especially underrepresented minorities, in his research. Currently, there are several undergraduate and M.S. graduate students including woman and minority students working in PI's lab, with support from AFOSR and NSF.

Collaboration with other scientists

During the last couple of years, the P.I. has been in closed contact/collaboration with theorists and applied mathematicians including Prof. Mark Ablowitz at Univ. of Colorado, Prof. D.N. Christodoulides at CREOL, University of Central Florida, Prof. J. Yang at University of Vermont, and Prof. N. K. Efremidis at University of Crete, Greece. Some of them are currently also AFOSR contractors in the nonlinear optics program. The P.I. will continue the collaboration with other contractors and discuss with them about productive plans and future collaboration for the AFOSR projects. 


\title{
Publications Acknowledged AFOSR Support during 2012-2015:
}

\author{
Book \& Book Chapters:
}

- Z. Chen, and R. Morandotti eds., Nonlinear Photonics and Novel Phenomena, Springer Series in Optical Sciences, Vol 171, ISBN 978-1-4614-3537-2 (Springer, 2012).

- Y. Hu, P. Zhang, Z. Chen and G. A. Siviloglo, D. N. Christodoulides, "Generation and control of accelerating Airy beams”, in Nonlinear Photonics and Novel Phenomena, Vol 171. Z. Chen, and R. Morandotti eds. (Springer, 2012).

- P. Zhang, Cibo Lou, Yi Hu, Sheng Liu, Jianlin Zhao, Jingjun Xu, and Zhigang Chen, "Spatial beam dynamics mediated by hybrid nonlinearity", in Nonlinear Photonics and Novel Phenomena, Vol 171. Z. Chen, and R. Morandotti eds. (Springer, 2012).

\section{Referred Journal Actricles:}

- P. Zhang, Yi Hu, D. Cannan*, A. Salandrino, T. Li, R. Morandotti, X. Zhang, and Z. Chen, "Generation of linear and nonlinear nonparaxial accelerating beams", Opt. Lett., 37, 2820 (2012).

- D. Song, C. Lou, L. Tang, Z. Ye, J. Xu and Z. Chen, "Experiments on linear and nonlinear localization of optical vortices in optically-induced photonic lattices”, International Journal of Optics, Invited Review Article, Article ID 273857, (2012).

- S. Liu, Y.Hu, P. Zhang, X. Gan, C.Lou, D. Song, J. Zhao, J. Xu, and Z. Chen, "Symmetrybreaking diffraction and dynamical self-trapping in optically induced hexagonal photonic lattices", Appl. Phys. Lett. 100, 061907 (2012).

- Chremmos, Z. Chen, D. N. Christodoulides, and N.K. Efremidis, "Abruptly autofocusing and autodefocusing optical beams with arbitrary caustics”, Phys. Rev. A, 85, 023828 (2012).

- S. Liu, Yi Hu, P. Zhang, X. Gan, C. Lou, D. Song, J. Zhao, J. Xu, and Z. Chen, “Tunable selfshifting Bloch modes in anisotropic hexagonal photonic lattices”, Opt. Lett., 37, 2184 (2012).

- Z. Zhang, D. Cannan*, P. Zhang, J. Liu, D.N. Christodoulides and Z. Chen, "Observation of trapping and transporting air-borne particles with a single optical beam”, Opt. Express, 20, 16212 (2012).

- J. Yang, D. Gallardo*, A. Miller* and Z. Chen, "Elimination of transverse instability in stripe solitons by one-dimensional lattices”, Opt. Lett., 37, 1571 (2012).

- Y. Hu, Z. Sun, D. Bongiovanni, D. Song, C. Lou, J. Xu, Z. Chen and R. Morandotti, "Reshaping the trajectory and spectrum of nonlinear Airy beams”, Opt. Lett., 37, 3201 (2012).

- P. Zhang, D. Hernandez*, D. Cannan*, Y. Hu, S. Fardad, S. Huang*, J. Chen, D. N. Christodoulides and Z. Chen, "Rotating beads and bacteria with optical tweezers based on propelling beams”, Biomedical Opt. Express, 3, 1892 (2012).

- Z. Chen, M. Segev, D.N. Christodoulides, "Optical spatial solitons: historical overview and recent advances”, Invited review paper, Rep. Prog. Phys. 75, 086401 (2012).

- P. Zhang, Y. Hu, T. Li, D. Cannan*, X. Yin, R. Morandotti, Z. Chen, and X. Zhang, “Nonparaxial Mathieu and Weber Accelerating Beams”, Phys. Rev. Lett. 109, 193901 (2012). (see "Synopsis: Optical Boomerangs", This week in Physics - November 19, 2012; "OPN Scatterings: Two New Classes of Accelerating Light Beams”, November 19, 2012).

- D. Chremmos, Z. Chen, D. N. Christodoulides and N. K. Efremidis, "Bessel-like optical beams with arbitrary trajectories”, Opt. Lett. 37, 5003 (2012).

- P. Zhang, D. Hernandez*, D. Cannan*, Y. Hu, S. Fardad, S. Huang*, J. Chen, D. N. Christodoulides and Z. Chen, "Rotating Beads and Bacteria with Moiré-based Optical Tweezers", Opt. \& Photonics News, December issue (“Optics in 2012”). 
- J. Zhao, P. Zhang, D. Deng, J. Liu, Y. Gao, I. D. Chremmos, N. K. Efremidis, D. N. Christodoulides, and Z. Chen, "Observation of self-accelerating Bessel-like optical beams along arbitrary trajectories," Opt. Lett. 38, 498-500 (2013).

- Y. Hu, M. Li, D. Bongiovanni, M. Clerici, J. Yao, Z. Chen, J. Azaña, and R. Morandotti, "Spectrum to distance mapping via nonlinear Airy pulses," Opt. Lett. 38, 380-382 (2013).

- Z. Zhang, P. Zhang, M. Mills, Z. Chen, D. N. Christodoulides, and J. Liu, "Trapping aerosols with optical bottle arrays generated through a superposition of multiple Airy beams," Chin. Opt. Lett. 11, 033502 (2013).

- M. C. Rechtsman, Y. Plotnik, J. M. Zeuner, D. Song, Z. Chen, A. Szameit and M. Segev, "Topological Creation and Destruction of Edge States in Photonic Graphene", Phys. Rev. Lett., 111, 103901 (2013).

- R. Driben, Y. Hu, Z. Chen, B. A. Malomed, and R. Morandotti, "Inversion and tight focusing of Airy pulses under the action of third-order dispersion," Opt. Lett. 38, 2499-2501 (2013).

- Y. Hu, D. Bongiovanni, Z. Chen and R. Morandotti, "Periodic self-accelerating beams by combined phase and amplitude modulation in the Fourier space," Opt. Lett. 38, 3387-3389 (2013).

- S. Fardad, M. S. Mills, P. Zhang. W. Man, Z. Chen, and D.N. Christodoulides, "Interactions between self-channeled optical beams in soft-matter systems with artificial nonlinearities”, Opt. Lett. 38, 3585 (2013).

- D. Deng, Y. Gao, J. Zhao, P. Zhang, and Z. Chen, "Three-dimensional nonparaxial beams in parabolic rotational coordinates," Opt. Lett. 38, 3934-3936 (2013).

- J. Zhao, P. Zhang, D. Deng, C. Lou, D. Song, J. Liu, and Z. Chen, "Self-accelerating and selfbreathing Bessel-like beams along arbitrary trajectories," Chin. Opt. Lett. 11, 110701 (2013).

- Y. Hu, D. Bongiovanni, Z. Chen and R. Morandotti, "Multi-path multi-component selfaccelerating beams through spectrum-engineered position mapping”, Phys. Rev. A, 88, 043809 (2013).

- W. Man, S. Fardad, Z. Zhang' J. Prakash, M. Lau*, P. Zhang, M. Heinrich, D. N. Christodoulides, and Z. Chen, "Engineered optical nonlinearities and enhanced light transmission in soft-matter systems with tunable polarizabilities”, Phys. Rev. Lett., 111, 218302 (2013).

- Z. Zhang, Z. Ye, D. Song, P. Zhang, Z. Chen, "Repositioning and steering laser beam power via coherent combination of multiple Airy beams”, Appl. Opt. 52, 8512 (2013).

- Y. Plotnik, M. C. Rechtsman, D. Song, M. Heinrich, J. M. Zeuner, S. Nolte, N. Malkova, J. Xu, A. Szameit, Z. Chen, and M. Segev, "Observation of unconventional edges states in photonic graphene”, Nature Material, 13, 57 (2014) (published online: 10 November 2013 । doi:10.1038/nmat3783)

- X. Qi, K.G. Makris, R. El-Ganainy, P. Zhang, J. Bai, D. N. Christodoulides, and Z. Chen, "Observation of accelerating Wannier-Stark beams in optically induced photonic lattices," Opt. Lett. 39, 1065-1068 (2014).

- K. G. Makris, I. Kaminer, R. El-Ganainy, N. K. Efremidis, Z. Chen, M. Segev, and D. N. Christodoulides, "Accelerating diffraction-free beams in photonic lattices," Opt. Lett. 39, 21292132 (2014).

- Y. Liang, Y. Hu, Z. Ye, D. Song, C. Lou, X. Zhang, J. Xu, R. Morandotti, and Z. Chen, "Dynamical 2D deformed Airy beams with arbitrary angles between two wings", J Opt Soc Am A 31(7):1468-72 (2014).

- S. Fardad, A. Salandrino, M. Heinrich, P. Zhang, Z. Chen and D. N. Christodoulides, "Plasmonic Resonant Solitons in Metallic Nanosuspensions”, Nano Letters 14, 2498-2504 (2014).

- V. Smith, B. Leung, P. Cala, Z. Chen, and W. Man, "Giant tunable self-defocusing nonlinearity and dark soliton attraction observed in m-cresol/nylon thermal solutions," Opt. Mater. Express 4, 1807-1812 (2014) 
- Y. Gao, D. Song, S. Chu, Z. Chen, “Artificial Graphene and Related Photonic Lattices Generated With a Simple Method”, IEEE Photonics Journal, accepted (2014).

- Y. Hu, A. Tehranchi, S. Wabnitz, R. Kashyap, Z. Chen, and R. Morandotti, "Improved IntraPulse Raman Scattering Control via Asymmetric Airy Pulses”, Phys. Rev. Lett. 114, 073901 (2015).

- V.Paltoglou, Z. Chen, and N. K. Efremidis, "Composite multi-vortex diffraction-free beams and van Hove singularities in honeycomb lattices”, Opt. Lett. 40, 1037 (2015).

- S. Xia, D. Song, Y. Zong, L. Tang, and Z. Chen, "Observation of self-trapping and rotation of higher-band gap solitons in two-dimensional photonic lattices", Opt. Express, 23, 4397-4405 (2015).

- D. Song, V. Paltoglou, S. Liu, Y. Zhu, D. Gallardo, L. Tang, J. Xu, M. Ablowitz, N. K. Efremidis, and Z. Chen, "Unveiling pseudospin and angular momentum in photonic graphene", Nature Communications, 6, Article number: 6272 (2015) doi:10.1038/ncomms7272 
1.

\section{Report Type}

Final Report

\section{Primary Contact E-mail}

Contact email if there is a problem with the report.

zhigang@sfsu.edu

Primary Contact Phone Number

Contact phone number if there is a problem with the report

4153383876

Organization / Institution name

San Francisco State Univ

\section{Grant/Contract Title}

The full title of the funded effort.

Light Control and Image Transmission through Photonic Lattices with Engineered Coupling

\section{Grant/Contract Number}

AFOSR assigned control number. It must begin with "FA9550" or "F49620" or "FA2386".

FA9550-12-1-0111

\section{Principal Investigator Name}

The full name of the principal investigator on the grant or contract.

\section{Zhigang Chen}

\section{Program Manager}

The AFOSR Program Manager currently assigned to the award

Arje Nachman

\section{Reporting Period Start Date}

04/15/2012

\section{Reporting Period End Date}

$03 / 31 / 2015$

\section{Abstract}

The objective of this project is to develop research programs in photonic lattices that are at the frontier of nonlinear optics/photonics for fundamental understandings in scientific knowledge as well as for possible applications of direct interest to the Air Force. The proposed studies include mainly beam control in engineered photonic lattices, Tamm and Shockley-like edge states and topological surface states in 2D honey-comb lattices ("photonic graphene"), and light localization and transport in disordered lattices. Apart from these proposed studies, the project made additional efforts on design and control of self-accelerating beams and nonlinear enhanced transmission in synthetic colloidal nanosuspensions. These studies will advance the knowledge in several interdisciplinary areas such as nonlinear optics, condensed matter physics, atmospheric sciences, and will also have many application potentials.

\section{Distribution Statement}

This is block 12 on the SF298 form.

Distribution A - Approved for Public Release 
If this is not approved for public release, please provide a short explanation. E.g., contains proprietary information.

\section{SF298 Form}

Please attach your SF298 form. A blank SF298 can be found here. Please do not password protect or secure the PDF The maximum file size for an SF298 is $50 \mathrm{MB}$.

$$
\text { AF_SF298_2015.pdf }
$$

Upload the Report Document. File must be a PDF. Please do not password protect or secure the PDF. The maximum file size for the Report Document is 50MB.

$$
\text { AFOSR_finalReport_March2015.pdf }
$$

Upload a Report Document, if any. The maximum file size for the Report Document is $50 \mathrm{MB}$.

Archival Publications (published) during reporting period:

See the list in the report

Changes in research objectives (if any):

Change in AFOSR Program Manager, if any:

Extensions granted or milestones slipped, if any:

AFOSR LRIR Number

LRIR Title

Reporting Period

Laboratory Task Manager

Program Officer

Research Objectives

Technical Summary

Funding Summary by Cost Category (by FY, \$K)

\begin{tabular}{|l|l|l|l|}
\hline & Starting FY & FY+1 & FY+2 \\
\hline Salary & & & \\
\hline Equipment/Facilities & & & \\
\hline Supplies & & & \\
\hline Total & & \\
\hline
\end{tabular}

Report Document

Report Document - Text Analysis

Report Document - Text Analysis

Appendix Documents

2. Thank You

E-mail user

Apr 23, 2015 00:51:30 Success: Email Sent to: zhigang@sfsu.edu 